\title{
Assessment of the Levels and Potential Health Risk Posed by Selected Organophosphate Pesticide Residues in Vegetable Farms in Manzini Region, Eswatini
}

\author{
Gabriel Chewe Bwembya ${ }^{1, ~ *}$, Paul Junior ${ }^{1}$, Justice Mandla Thwala ${ }^{1}$, Sipho Samuel Mamba ${ }^{1}$, \\ Tesfamariam Yosief Debessai ${ }^{1}$, Nomfundo Dlamini ${ }^{2}$ \\ ${ }^{1}$ Department of Chemistry, Faculty of Science and Engineering, University of Eswatini, Kwaluseni, Eswatini \\ ${ }^{2}$ Department of Food Science, Nutrition and Technology, Faculty of Agriculture, University of Nairobi, Kabete, Kenya
}

Email address:

gbwembya@uniswa.sz (G. C. Bwembya)

${ }^{*}$ Corresponding author

\section{To cite this article:}

Gabriel Chewe Bwembya, Paul Junior, Justice Mandla Thwala, Sipho Samuel Mamba, Tesfamariam Yosief Debessai, Nomfundo Dlamini. Assessment of the Levels and Potential Health Risk Posed by Selected Organophosphate Pesticide Residues in Vegetable Farms in Manzini Region, Eswatini. International Journal of Bioorganic Chemistry. Vol. 4, No. 1, 2019, pp. 53-63. doi: 10.11648/j.ijbc.20190401.18

Received: February 18, 2019; Accepted: March 30, 2019; Published: May 6, 2019

\begin{abstract}
The study investigated qualitatively and quantitatively the presence of organophosphate pesticide (OPP) residues in cabbages, tomatoes and soil from vegetable gardens of Manzini region and assessed the health risk to consumers. Samples were analysed using QuEChERS extraction technique and GC-ECD. Mean recoveries of the pesticides range from $70.61 \%$ to $103.76 \%$. Among 26 samples of cabbages analysed, 8 samples $(30.77 \%)$ contained residues of dichlorvos with a mean concentration of $2.889 \pm 0.617 \mathrm{mg} / \mathrm{kg}, 8$ samples (30.77\%) contained residues of dimethoate with mean concentration of 2.898 $\pm 0.894 \mathrm{mg} / \mathrm{kg}$ while 5 samples $(19.23 \%)$ contained both dichlorvos and dimethoate. The highest concentration of dichlorvos of $5.460 \pm 0.911 \mathrm{mg} / \mathrm{kg}$ was found in Ngwempisi cabbages while the lowest concentration of $0.415 \mathrm{mg} / \mathrm{kg}$ was in Mgazini cabbages. The highest amount of dimethoate of $13.150 \pm 3.246 \mathrm{mg} / \mathrm{kg}$ was in Matfunjwa cabbages while the lowest amount of dimethoate of $0.106 \pm 0.187 \mathrm{mg} / \mathrm{kg}$ was in Boyane cabbages. All the contaminated cabbages had residue levels above the Codex (FAO/WHO) and EU MRLs. None of the 15 samples of tomatoes was found to be contaminated with organophosphate pesticides. Out of 41 samples of soil, chlorpyrifos was detected in only 3 samples $(7.32 \%)$ with mean concentration of $1.145 \pm$ $0.136 \mathrm{mg} / \mathrm{kg}$. The highest amount of $2.778 \pm 0.134 \mathrm{mg} / \mathrm{kg}$ was found in soil from Buoyane. The health risk estimate also demonstrated that the levels of pesticides residues in cabbages pose health threat to consumers. The results of this study provide a database on the levels of organophosphate pesticide residues in vegetables in the Manzini region to the Ministries of Agriculture and Health. This will help introduce safer pesticide management practices. It will also bring awareness to the general public on the dangers of OPPs to human health and environment.
\end{abstract}

Keywords: Health-Risk, GC-ECD, Organophosphate Pesticides, QuEChERS, Vegetables

\section{Introduction}

Vegetables are rich in minerals and notable diets of Emaswati people. However, because of pest and insect infestation, they are preserved with pesticides by farmers. Pesticides include chemical substances used to kill animals, plants and insects in agricultural and domestic settings such as herbicides, insecticides, fumigants, rodenticides and fungicides with different mechanisms [1]. Major classes of pesticides include; organochlorine, organophosphates, carbamates and pyrethroids. After the cessation of use of organochlorine insecticides, organophosphate pesticides (OPPs) which are derivatives of phosphoric acid became the most commonly used pesticides. Organophosphate pesticides were originally developed in the 1940 s as highly toxic biological warfare agents referred to as Nerve Agents [2]. 
OPPs affect the nervous system by disrupting the enzyme that regulates acetylcholine a neural transmitter substance leading to elevated levels of acetylcholine and hence acute and chronic effects [3]. OPPs can be fairly persistent which may cause threat to human beings, aquatic organisms and birds due to their high toxicity.

Organophosphorus pesticide self-poisoning is an important clinical problem in rural regions of the developing world, and kills nearly 200000 people every year. Unintentional poisoning kills far fewer people but is a problem in places where highly toxic organophosphorus pesticides are available [4]. According to the World Bank [5] nearly 355,000 people die worldwide each year from unintentional pesticide poisoning. Hence, there is need to monitor the levels of these pesticides in food and the environment.

The OPPs enter the body through all routes such as via; Dermal exposure hence subsequent absorption through the intact skin, Oral route through accidental ingestion or consumption of contaminated food, Inhalation during spraying and also through mucous membranes and eyes [6]. However, exposure of human to pesticides is mainly from residues in food, where the level of exposure depends on both the quantity of food consumed and the level of residues [7]. Therefore, presence of pesticides in food has health implications.

Akan and co-workers [8] investigated the presence of organophosphorus pesticide residues in some vegetables and soil samples from Alau Dam and Gongulong agricultural areas in Borno State, Nigeria and found that the concentrations of OPPs were at alarming levels, much higher than the maximum residue limits (MRLs) and acceptable daily intake values (ADIs) set for vegetables and soil by the European Union (EU). Hence, the need for continuous monitoring is recommended so as to regulate the use of pesticides. Twenty-one pesticide residues were detected in cabbages from farm gate in Cape Coast, Ghana and nine of these were pyrethroids and twelve were organophosphates [9]. The conclusion from this study was that the high levels and wide assortment of pesticide residues found suggest the need to put into effect regulations regarding proper use of pesticides on vegetables.

Eswatini like other developing countries, in order to improve income and provide gainful employment, diversification from grain crops to high value crops like vegetables has appeared to be an essential strategy for agricultural growth [10]. The agricultural sector in Eswatini is characterised by a dualistic system with modern and traditional sectors. About $80 \%$ of the Swazi population lives on Swazi Nation Land (SNL) and obtain their livelihood from subsistence agricultural production activities [11]. The Ministry of Agriculture encourages the intensification of agricultural production amongst small-scale farmers on SNL. Although the performance of large farms and plantations on Title Deed Land (TDL) remains crucial to export growth and overall economic development in Eswatini, increasing rural employment and income depends mainly on the performance of agriculture on SNL. Accordingly, the Eswatini government focuses its resources on SNL, whilst ensuring that support does not negatively affect agricultural production on TDL. However, the rate of adoption of sugarcane production on SNL is threatening the production of irrigated crops, especially vegetables [11]. Vegetables by smallholder farmers are mainly produced for consumption and are also sold if there is a surplus. Between 2005 and 2009, a total of 559 farmers were involved in production of vegetables such as tomatoes, cabbages, carrots and onions under a total area of 353.7 hectares, with a total contribution of E 278,481 to gross domestic product of Eswatini [12]. FAOSTAT [13] reported that Eswatini's total vegetable production from 2010 to 2012 was ranging from 12,450 to 13,000 metric tonnes. Most vegetable farmers around Manzini region use organophosphate pesticides during the production of vegetables to control and eliminate parasites, insects and fungal diseases in order to meet the demand for quantity and quality of their agricultural products and this result in entry of chemical contaminants into vegetable farms leading to pollution of soils, crops, rivers and air. Pesticides can remain as residues in soils, crop surfaces or water from where human beings are constantly exposed [14]. Depending on the type of pesticide applied, the vapours and residues may also drift or volatilize off the treatment site thereby contaminating air, soil and vegetation and increasing the potential for human and animal exposure. As a result of extensive use of chemicals, there is need to determine the levels of pesticides in vegetable farms in Eswatini.

Even though OPPs are commonly used in Eswatini, there are no previous studies that have been done in Eswatini to establish the extent of contamination of vegetable fields with organophosphate pesticide residues. Hence, there is a need to carry out thorough investigation on these as they are highly toxic to humans and animals as well as the soil where vegetables are grown. Also in most developing countries like Eswatini there are no strict control measures to regulate the use of pesticides. Although importation and licensing of pesticides is usually regulated by the government, selling and buying is often not controlled such that consumers are free to buy and use pesticides at will. Pesticides residues screening on foods is often not mandatory. In such a situation researches dedicated to determine pesticide residues levels in such products become most crucial thereby providing the rationale behind this study.

Therefore, this study was done to help bridge information gap in pesticide research in Eswatini. It will also provide information on the different organophosphate pesticides used in vegetable fields in Eswatini and enable scientific community assess the extent of contamination of vegetables sold in the markets. It will also guide policy makers in instituting measures to protect vegetable consumers and also regulate pesticides in the country as well as formulate laws concerning pesticides.

This work aimed at: (i) determining the concentration of selected organophosphate pesticide residues (chlorpyrifos, dimethoate, malathion, profenofos and dichlorvos) in soil samples from vegetable farms around Manzini region; (ii) 
determining the concentration of organophosphate pesticide residues in tomatoes and cabbages and (iii) assessing the potential health risks resulting from exposure to these pesticides via dietary intake. Furthermore, the results were compared with recommended European Union (EU) maximum residue limits (MRLs) [15] and the acceptable daily intake values set by Food and Agriculture Organisation (FAO)/World Health Organisation (WHO) Codex Alimentarius Commission [16].

\section{Materials and Methods}

\subsection{Study Area}

The study was conducted in the vegetable growing areas in Manzini which is a region of Eswatini located in the centerwest of the country. It has an area of $4,093.59 \mathrm{~km}^{2}$ and a population of 319,530 in 2015 and is divided into 16 tinkhundla [17]. Its administrative centre is Manzini. It borders all three other regions: Hhohho in the north, Lubombo in the east, and Shiselweni in the south. It is bordered by the Mpumalanga province in South Africa to the west [17]. It covers Highveld, middleveld and down to the lowveld. The climate here is mild, and generally warm and temperate. The rainfall in Manzini averages $881 \mathrm{~mm}$ with precipitation even during the driest months. The average temperature is about $19.6{ }^{\circ} \mathrm{C}$. These conditions are favourable for vegetable production. The study area and the sampling sites are shown in figures 1 and 2 respectively.

\subsection{Sampling of Vegetables}

The sampling of vegetables was performed in accordance with the general principles and methods of the European Commission (EC) directive 2002/63/EC [18] for establishing MRLs in food commodities. Each representative vegetable sample was a composite of 10 subsamples of the same commodity collected through random sampling. All the samples (1-2 kg each) were collected from fifteen (15) vegetable schemes using gloves and placed in black plastic bags in an ice chess box to avoid contamination and deterioration, labelled and transported to the laboratory and preserved in a refrigerator at below $4^{\circ} \mathrm{C}$, pending extraction. A total of 150 tomatoes were collected from different gardens and put in groups of 10 to make 15 composite samples of tomatoes and 26 cabbage samples were sampled with at least 3 cabbages taken from each garden to minimise on costs. A total of 41 composite vegetable samples were collected.

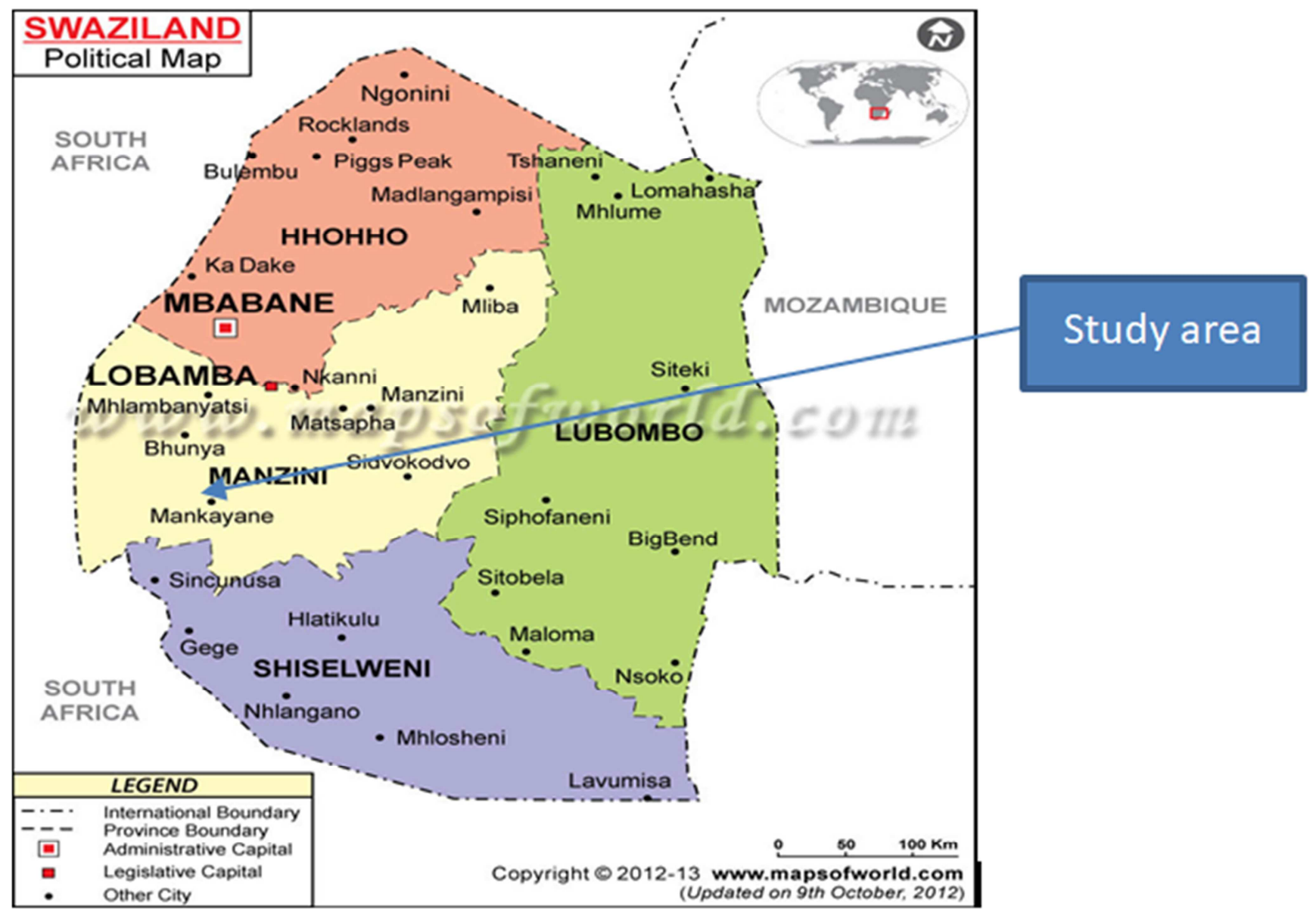

Source: (https://www.mapsofworld.com/swaziland/swaziland-political-map.html)

Figure 1. Map of Eswatini showing the study area. 


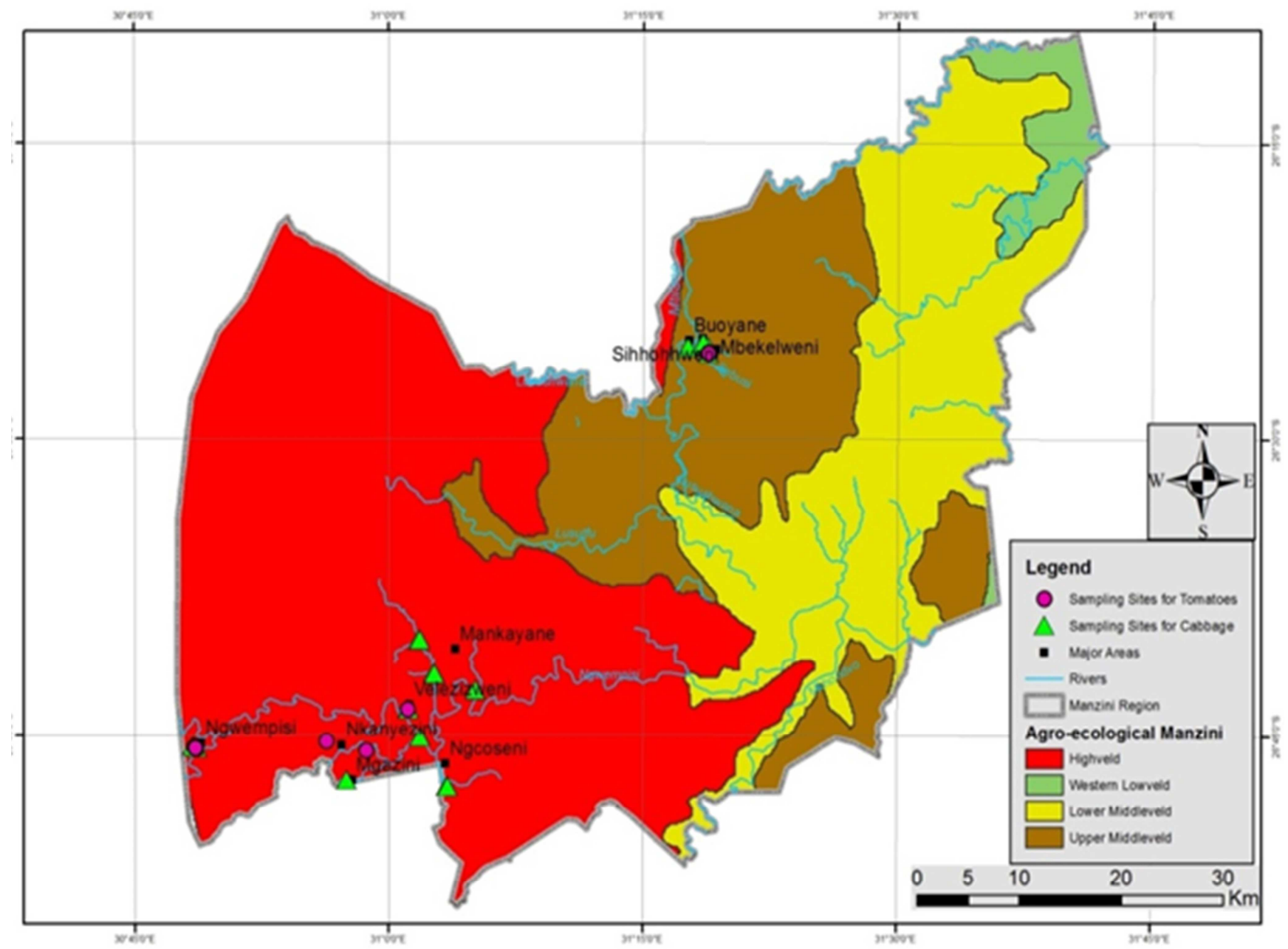

Figure 2. Sampling areas in the Manzini region of Eswatini.

\subsection{Sampling of Soil Samples}

In each vegetable farm, soil samples were taken at 5 random points at a depth of $0-20 \mathrm{~cm}$ and mixed to form a composite $0.5-1 \mathrm{~kg}$ sample. Soil sampling was done using a soil auger and samples were packed in black plastic sampling bags to avoid photochemical oxidation, labelled and transported to the laboratory, kept refrigerated at below $4^{\circ} \mathrm{C}$ away from light prior to extraction. A total of 41 soil samples were collected. Also during sampling, exposure of samples to dust was minimized to prevent contamination of samples since this was going to negatively affect the results.

\subsection{Pre-Treatment of Vegetable and Soil Samples}

The vegetable samples were chopped into small pieces and blended using a blender. The blender was cleaned thoroughly with soap and deionised water and dried before being used for next sample to avoid cross contamination. A representative portion $(10.0 \pm 0.1 \mathrm{~g})$ was then weighed and taken through extraction and clean up steps.

Prior to extraction, soil samples were air dried in dust free cabins at room temperature, ground to small particle sizes using a mortar and pestle, homogenised and sieved through a $500 \mu \mathrm{m}$ sieve to generate samples with a large surface area. A representative portion $(3.0 \pm 0.1 \mathrm{~g})$ was then weighed and taken for extraction.

\subsection{Chemicals and Reagents}

All reagents used for analysis of pesticide residues were of analytical grade. Pesticide reference standards of chlorpyrifos (purity $\geq 99.3 \%$ ), malathion (purity $\geq 99.2 \%$ ), dimethoate (purity $\geq 99.8 \%$ ), dichlorvos (purity $\geq 99.8 \%$ ) and profenofos (purity $\geq 97.6 \%$ ) were obtained from Sigma-Aldrich and QuEChERS kits containing magnesium sulphate $\left(\mathrm{MgSO}_{4}\right)$, sodium chloride $(\mathrm{NaCl})$, Primary Secondary Amine (PSA) and acetonitrile (HPLC grade) were supplied by Chemoquip distributors, South Africa.

\subsection{Preparation of Pesticide Standard Solutions}

Pesticide standard stock solutions of chlorpyrifos, dimethoate, malathion, profenofos and dichlorvos were prepared separately in acetonitrile at a concentration of 1000 $\mathrm{mg} / \mathrm{L}$ and stored at below $4^{\circ} \mathrm{C}$ away from light until use. A mixed standard solution of $50 \mathrm{mg} / \mathrm{L}$ in acetonitrile containing the aforementioned pesticides was prepared by adding the appropriate volume of each individual stock solution in a 50 $\mathrm{mL}$ volumetric flask and made to volume by addition of 
acetonitrile. An intermediate mixed standard solution of 10 $\mathrm{mg} / \mathrm{L}$ in acetonitrile was prepared from the mixed standard solution of $50 \mathrm{mg} / \mathrm{L}$. Then working standard solutions of 0.5 , $1.0,2.0,3.0$ and $5.0 \mathrm{mg} / \mathrm{L}$ in acetonitrile were prepared by transferring the appropriate amount from $10 \mathrm{mg} / \mathrm{L}$ intermediate mixed standard solution into separate $10-\mathrm{mL}$ volumetric flasks. All the standard solutions were kept in a refrigerator at below $4^{\circ} \mathrm{C}$ until use.

\subsection{Extraction and Clean Up of Vegetable and Soil Samples}

QuEChERS extraction technique based on Anastassiades and co-workers [19] and modified by Prodhan and coresearchers. [20] was used for the extraction and clean-up of samples. The chopped vegetable samples were ground thoroughly with the fruit blender. A representative $10.0 \pm 0.1$ $\mathrm{g}$ portion of thoroughly homogenized sample was then weighed in a $50 \mathrm{~mL}$ polypropylene centrifuge tube. Then 10 $\mathrm{mL}$ of acetonitrile $(\mathrm{MeCN})$ was added into the centrifuge tube. The centrifuge tube was closed properly and shaken vigorously for $30 \mathrm{~s}$ by hand. After which, $4 \mathrm{~g}$ of anhydrous $\mathrm{MgSO}_{4}$ and $1 \mathrm{~g}$ of $\mathrm{NaCl}$ were added into the centrifuge tube, and shaken immediately by hand for 1 minute to prevent the formation of magnesium sulphate aggregates. Afterwards, the extract was centrifuged for $5 \mathrm{~min}$ at $4000 \mathrm{rpm}$. An aliquot of $6 \mathrm{~mL}$ of the MeCN layer (upper layer) was transferred into a $15 \mathrm{~mL}$ micro centrifuge tube containing $900 \mathrm{mg}$ anhydrous $\mathrm{MgSO}_{4}$ and $150 \mathrm{mg}$ Primary Secondary Amine (PSA). Then the mixture was thoroughly mixed by hand for $30 \mathrm{~s}$ and centrifuged for 5 minutes at $4000 \mathrm{rpm}$. After centrifuge, $1 \mathrm{~mL}$ of clean extract was filtered using a $0.45 \mu \mathrm{m}$ PTFE filter, and then added to a clean GC vial for injection. Triplicate extractions were carried out for each sample.

$3.0 \pm 0.1 \mathrm{~g}$ portions of well dried, sieved and homogenized soil samples was weighed in a $50 \mathrm{~mL}$ polypropylene centrifuge tube and $7 \mathrm{~mL}$ of deionised water was added and allowed to hydrate. Then $10 \mathrm{~mL}$ of acetonitrile was added and the mixtures taken through the procedures described above for extraction and clean up of vegetable samples.

\subsection{Identification and Quantification of Pesticide Residues in the Samples}

The clean extracts were subjected to analysis by Shimadzu 2010 plus Gas Chromatography (GC) equipped with an Electron Capture Detector (ECD). The identification of selected pesticides was performed by comparing peak retention times in samples to those of peaks in the pure analytical standards and quantification by use of area under the peaks. The GC conditions were as follows; injector temperature: $250^{\circ} \mathrm{C}$; detector temperature: $300^{\circ} \mathrm{C}$; carrier gas nitrogen $\left(\mathrm{N}_{2}\right)$, optimum flow rate of $0.6 \mathrm{~mL} / \mathrm{min}$; make up, flow rate of $30 \mathrm{~mL} / \mathrm{min}$; column temperature program: initially $150^{\circ} \mathrm{C}$ hold for 1 minute, increase at $5^{\circ} \mathrm{C} / \mathrm{min}$ to $240^{\circ} \mathrm{C}$, hold for 5 minutes; injection volume: $1 \mu \mathrm{L}$, split ratio, 1:10. Total run time was 24 minutes. Shimadzu, GC solution software was used for instrument control and data analysis.

\subsection{Analytical Quality Control}

Quality control and quality assurance were also incorporated in the analysis scheme. The accuracy and precision was evaluated through recovery studies and linearity was evaluated at five calibration levels using external standards. Limit of detection and limit of quantification were assessed based on the lowest concentration of residues that could be reproducibly measured at the operating conditions of GC. Blank and spike analyses were also carried out on every batch of samples in order to check any interfering species in the reagents.

\subsection{Target Hazard Quotient (THQ) Estimation}

Target hazard quotient (THQ) was estimated in accordance with the U.S. EPA [21] standard method of estimating the risk of non-carcinogenic effects. The dose calculations were carried out using the standard assumption from an integrated U.S. EPA risk analysis. An average weight of adults was considered as $70 \mathrm{~kg}[22,23]$. It was also based on assumption that processing such as cooking, boiling etc. does not have effect on the levels of contaminants and the absorption of each contaminant is $100 \%$. The THQ was determined based on the method by Chien and co-workers [24] as described by equation (1):

$$
T H Q=\frac{F r \times D e x \times I r \times C}{R f d \times B w \times T a} \times 10^{-3}
$$

Where $T H Q$ is the target hazard quotient; $F r$ is the exposure frequency (365 days per year); Dex is the exposure duration (52 years); equivalent to the average lifetime; $I r$ is the food consumption rate (g/person/day). Food consumption rate for vegetables in Eswatini is $40 \mathrm{~kg} /$ person/year [25] which is approximately $0.1096 \mathrm{~kg} /$ person/day; $C$ is the pesticide concentration in the food $(\mu \mathrm{g} / \mathrm{g}) ; B w$ is the average body weight $70 \mathrm{~kg}$ for adults; $R f d$ is the reference dose which are considered to be safe levels of exposure over the life time (U.S. EPA, 1996) and Ta is the average exposure time for non-carcinogens (365 days/ year $\mathrm{X}$ number of exposure years, assuming 52 years in this study).

\section{Results and Discussion}

\subsection{Calibration Curves and Linearity for Different Organophosphate Pesticides}

The concentration and peak area were used to prepare calibration curves for each analyte as shown in figures 3,4 , 5, 6 and 7 for dichlorvos, dimethoate, chlorpyrifos, malathion and profenofos respectively by using linear regression to obtain the equation of the standard curves for the tested pesticides. Linearity was evaluated by calculating the correlation coefficient, intercept and slope of the regression line at five concentration levels. The coefficients of determination were $\geq 0.98$ for all selected pesticides hence good linearity and reproducibility was achieved. The equations of the regression lines showing slope and intercept and the correlation coefficients are summarised in table 1. 


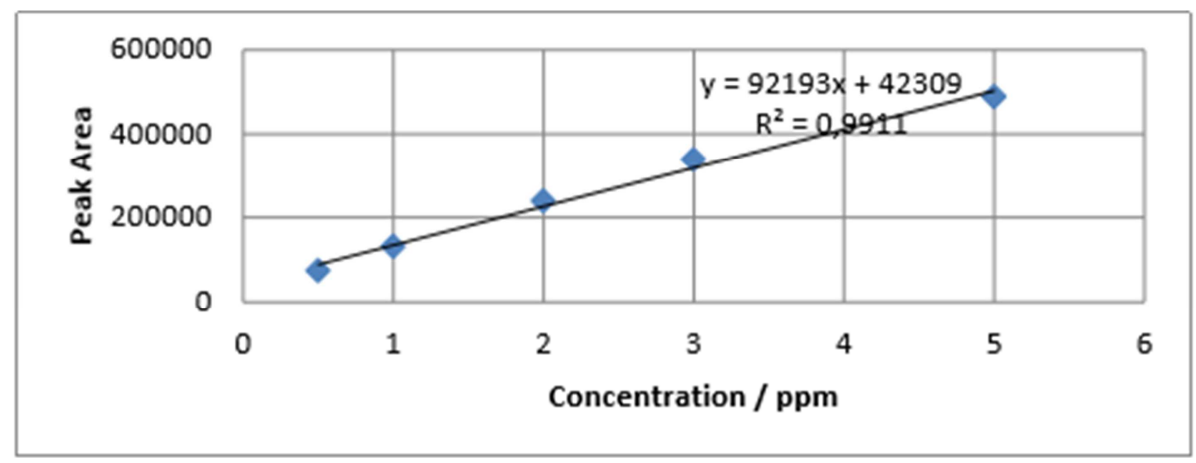

Figure 3. Calibration curve for Dichlorvos.

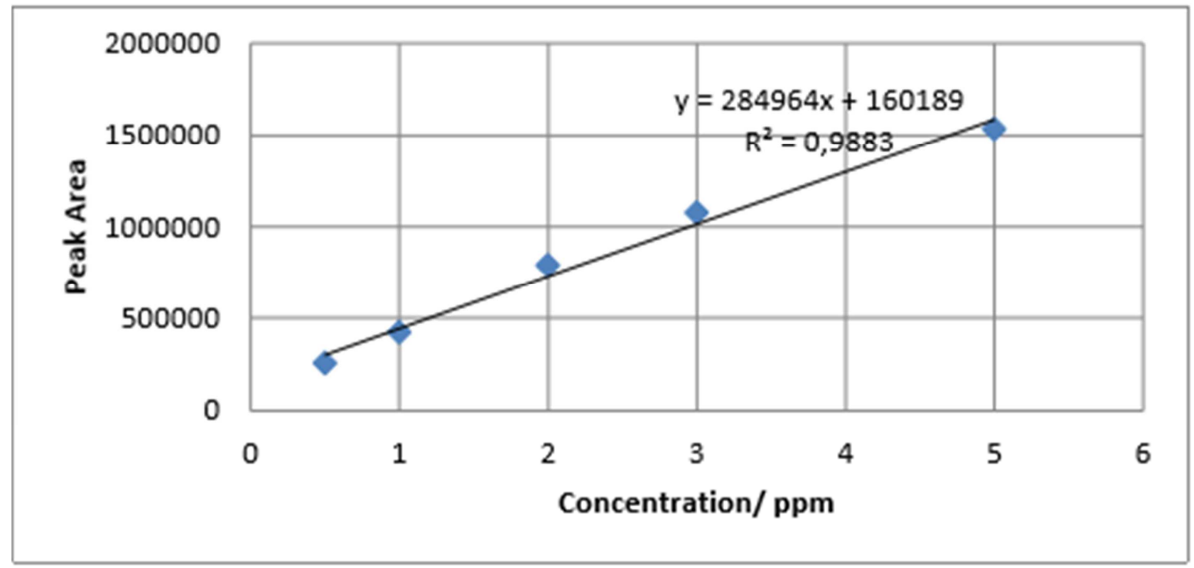

Figure 4. Calibration curve for Dimethoate.

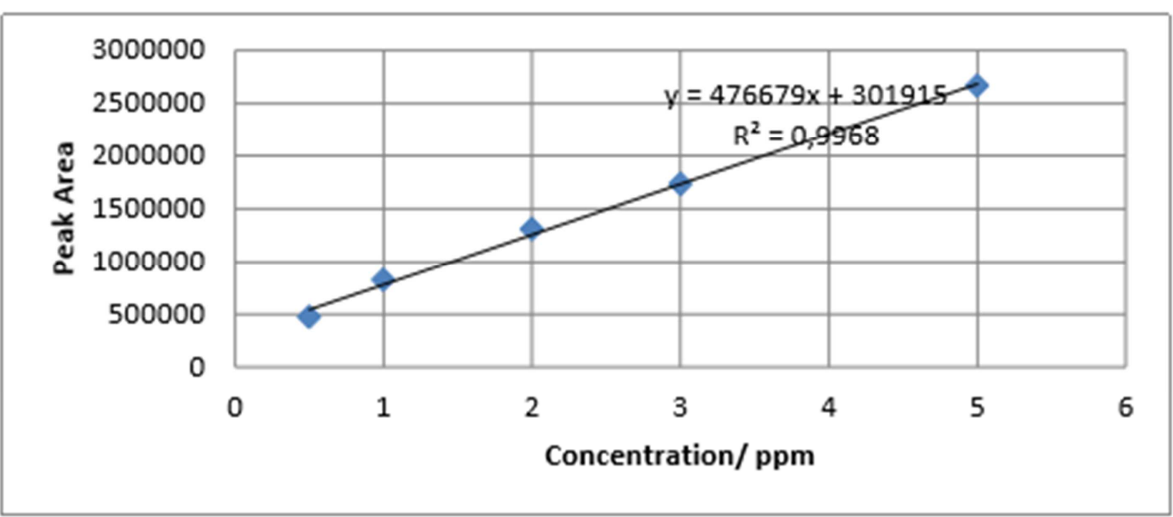

Figure 5. Calibration curve for Chlorpyrifos.

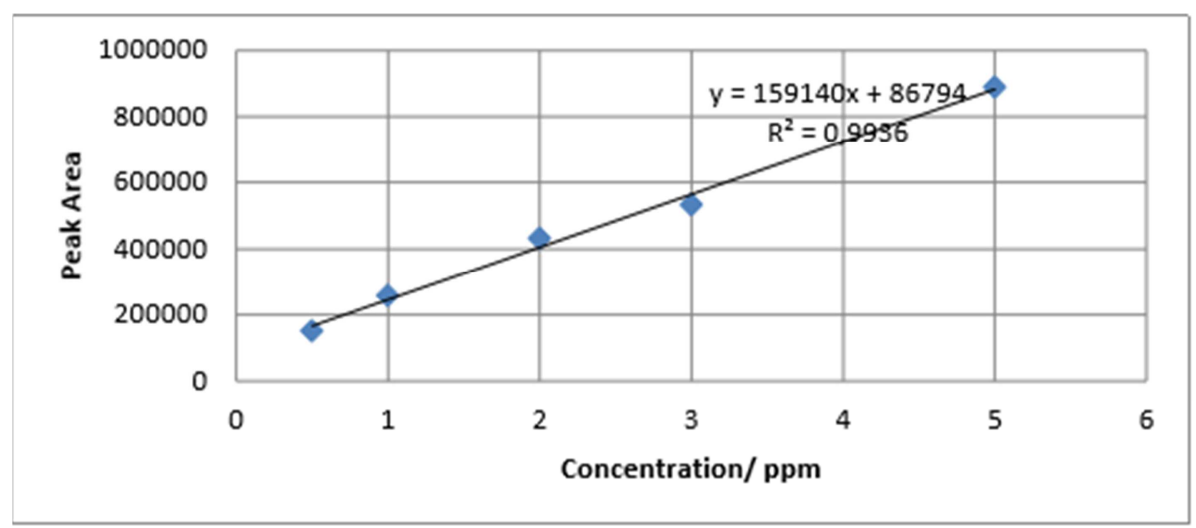

Figure 6. Calibration curve for Malathion. 


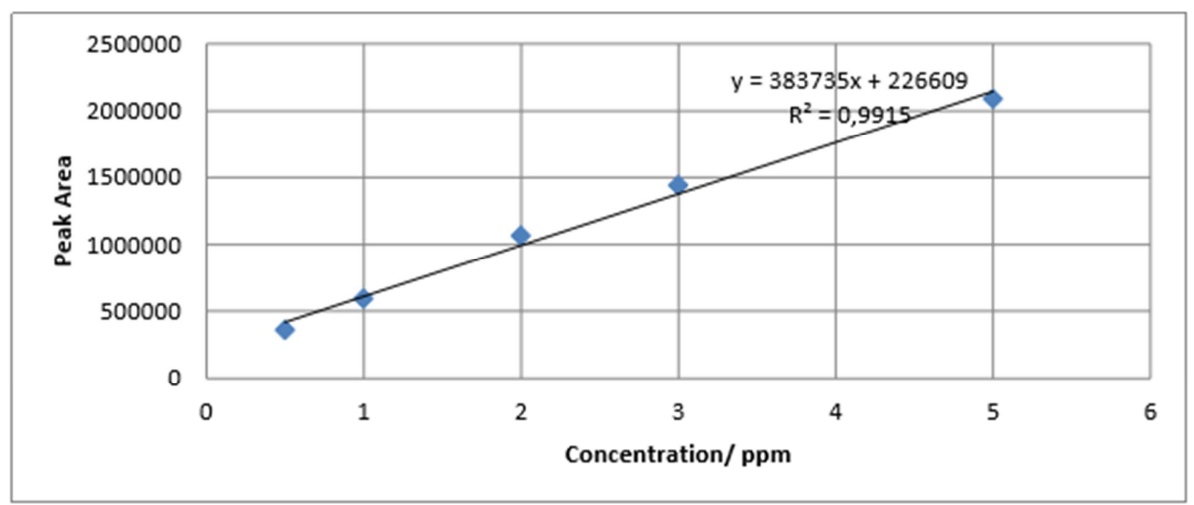

Figure 7. Calibration curve for Profenofos.

Table 1. Summary of calibration parameters for organophosphate pesticides.

\begin{tabular}{lll}
\hline Pesticides & Regression equation & $\mathbf{R}^{2}$ \\
\hline Dichlorvos & $\mathrm{y}=92193 \mathrm{x}+42309$ & 0.9911 \\
Dimethoate & $\mathrm{y}=284964 \mathrm{x}+160189$ & 0.9883 \\
Chlorpyrifos & $\mathrm{y}=476679 \mathrm{x}+301915$ & 0.9968 \\
Malathion & $\mathrm{y}=159140 \mathrm{x}+86794$ & 0.9936 \\
Profenofos & $\mathrm{y}=383735 \mathrm{x}+226609$ & 0.9915 \\
\hline
\end{tabular}

The chromatograms of the implicated pesticide standards and samples are shown in figures 8 and 9 respectively.

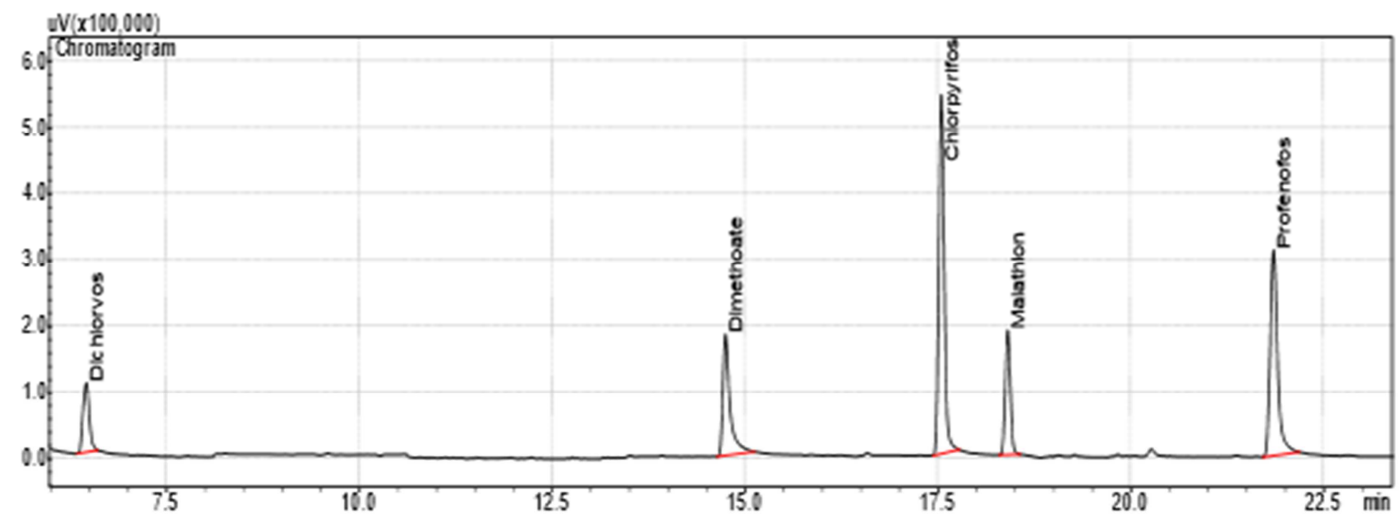

Figure 8. A typical GC Chromatogram of standards of organophosphate pesticides.

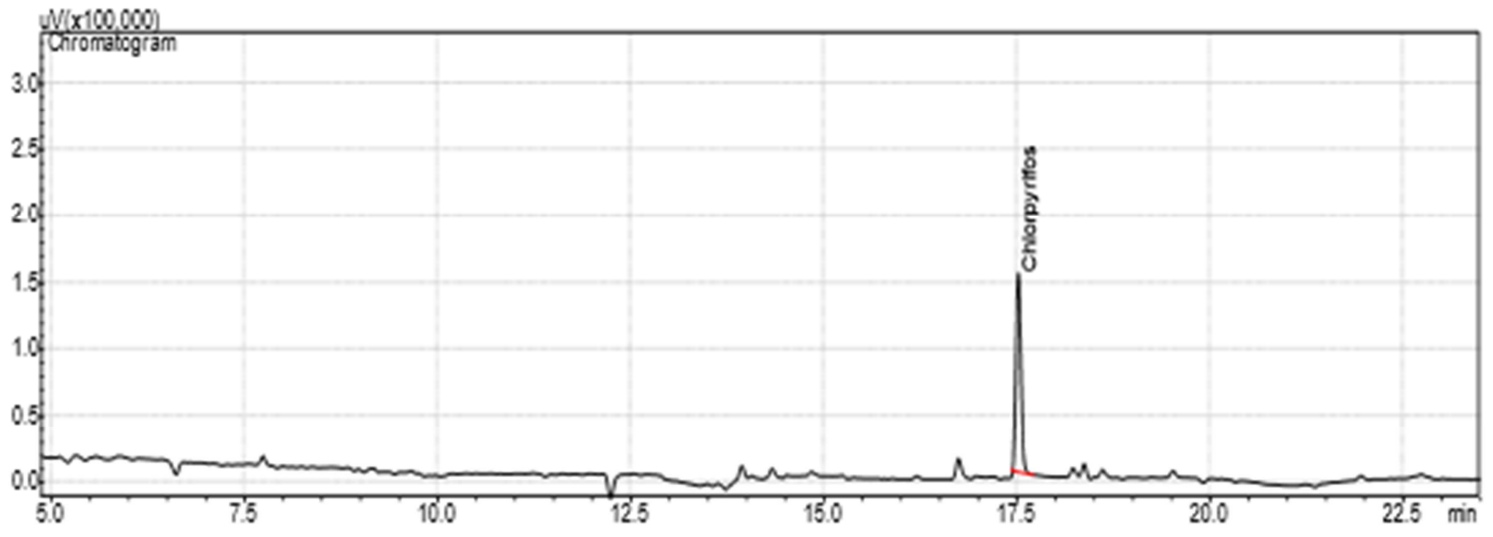

Figure 9. A typical GC chromatogram of soil sample contaminated with chlorpyrifos.

\subsection{Recovery}

Accuracy and precision of the method in terms of percentage recovery was determined for replicates of high spiked sample and low spiked sample analysed using the candidate method and recovery was estimated. The recoveries for all OPPs analysed range from $70.61 \%$ to $103.76 \%$ with percentage relative standard deviation $<20 \%$ as shown in table 2 . 
Table 2. Recoveries of selected OPPs in soil, cabbage and tomatoes.

\begin{tabular}{llll}
\hline \multirow{2}{*}{ Pesticide } & \% Mean Recoveries \pm RSD $(\mathbf{n}=\mathbf{6})$ \\
\cline { 2 - 4 } & Soil & Cabbage & Tomatoes \\
\hline Dichlorvos & $86.17 \pm 14.82$ & $88.90 \pm 2.49$ & $79.78 \pm 3.58$ \\
Dimethoate & $92.08 \pm 2.19$ & $81.20 \pm 15.46$ & $86.99 \pm 10.98$ \\
Chlorpyrifos & $78.07 \pm 1.46$ & $103.76 \pm 4.24$ & $70.61 \pm 11.74$ \\
Malathion & $84.18 \pm 19.23$ & $71.40 \pm 11.37$ & $75.85 \pm 5.84$ \\
Profenofos & $72.48 \pm 7.84$ & $95.49 \pm 19.05$ & $71.90 \pm 3.51$ \\
\hline
\end{tabular}

\subsection{Instrument Limit of Detection and Limit of Quantification}

The instrument LOD and LOQ were determined using EURACHEM guidelines [26]. The results are presented in table 3.

Table 3. Values for Instrument Limit of Detection and Limit of Quantification.

\begin{tabular}{lll}
\hline Pesticide & LOD & LOQ \\
\hline Dichlorvos & 0.289 & 0.963 \\
Dimethoate & 0.181 & 0.603 \\
Chlorpyrifos & 0.199 & 0.665 \\
Malathion & 0.207 & 0.670 \\
Profenofos & 0.167 & 0.557 \\
\hline
\end{tabular}

The instrument detection limit (IDL) is higher method detection limit (MDL) because in the determination of MDL, the sample preparation involves isolation and recovery of analytes into an extract from the original sample, hence a concentration factor as well as the efficiency of extraction, as defined by percentage recovery is involved. Thus, the MDL will be lower than IDL.

\subsection{Method Limit of Detection (LOD) and Limit of Quantification (LOQ)}

Limit of detection is the lowest amount of analyte in a sample that can be detected but not necessarily quantitated as an exact value while limit of quantification is the lowest amount of analyte in the sample that can be quantitatively determined with suitable precision and accuracy and in analytical procedures that exhibit baseline noise, the LOD is based on signal-to-noise ratio of $3: 1$ and LOQ can be based on the signal to noise (SNR) ratio of 10:1. In this study, the method detection limits were determined according to EURACHEM guidelines [26] where LOD and LOQ are obtained from standard deviation of 10 replicate analyses of a sample spiked at low concentration. The results for different organophosphate pesticides analysed are presented in table 4 .
Table 4. Values for Method Limit of Detection and Limit of Quantification

\begin{tabular}{lll}
\hline Pesticide & LOD & LOQ \\
\hline Dichlorvos & 0.044 & 0.145 \\
Dimethoate & 0.005 & 0.015 \\
Chlorpyrifos & 0.104 & 0.348 \\
Malathion & 0.119 & 0.398 \\
Profenofos & 0.167 & 0.557 \\
\hline
\end{tabular}

\subsection{Concentration of Organophosphate Pesticide Residues in Vegetables}

Analysis of selected vegetable samples (cabbages and tomatoes) from the Manzini region of Eswatini was performed to determine if they contain residues of organophosphate pesticides above the Maximum Residue Limits set by EU and FAO/WHO $[15,16]$ given in table 5 . Out of the pesticides analysed, only dichlorvos and dimethoate were detected in cabbage samples, none of the pesticides analysed was found in the 15 composite tomato samples from Manzini region. It is evident from tables 6 and 7 that all the cabbage samples that were detected by the instrument had concentrations of dichlorvos and dimethoate above the established EU and FAO/WHO MRLs [15. 16]. Of the 26 cabbage samples analysed, 8 samples $(30.77 \%)$ were found to be contaminated with dichlorvos with mean concentration of $2.889 \pm 0.617$ $\mathrm{mg} / \mathrm{kg}$. The highest concentration of dichlorvos of $5.460 \pm 0.911$ $\mathrm{mg} / \mathrm{kg}$ was found in cabbage sample collected from Ngwempisi farm (NGWC6) and the lowest concentration of $0.415 \mathrm{mg} / \mathrm{kg}$ was obtained in cabbage from Manzini (MGAC26) as shown in table 6. Dimethoate was found in 8 cabbage samples (30.77\%) with a mean concentration of $2.898 \pm 0.894 \mathrm{mg} / \mathrm{kg}$. The highest amount of dimethoate of $13.150 \pm 3.246 \mathrm{mg} / \mathrm{kg}$ was found in cabbage collected from Matfunjwa (MATC4) while the lowest amount of dimethoate of $0.106 \pm 0.187 \mathrm{mg} / \mathrm{kg}$ was found in cabbage collected from Boyane as illustrated in table 7. The differences in concentration of organophosphate pesticide residues in samples from different locations may be due to use of different concentrations depending upon the pest density, number of applications and inappropriate methods of application. Chlorpyrifos, malathion and profenofos were not detected in any of the vegetable samples collected. Dimethoate was the most common detected organophosphate pesticide because it is the most frequently used pesticide. Although malathion is also commonly used, it was not detected in any of the samples possibly due to its rapid degradation on both vegetation and soils. Five cabbage samples (19.23\%) were contaminated with both dichlorvos and dimethoate pesticides which may cause synergistic effects to the consumers.

Table 5. Different Maximum Residual levels.

\begin{tabular}{lllll}
\hline Cabbages & Tomatoes & \\
\hline Pesticide & Codex MRL (mg/kg) & EU MRL (mg/kg) & Codex MRL (mg/kg) \\
\hline Dichlorvos & - & 0.01 & - & EU MRL (mg/kg) \\
Dimethoate & 0.05 & 0.01 & - & 0.01 \\
Chlorpyrifos & 1 & 0.05 & - & 0.01 \\
Malathion & - & 0.01 & 0.5 & 0.01 \\
Profenofos & 10 & 0.01 & 10 & 0.01 \\
\hline
\end{tabular}

Source: Codex Alimentarius International Food Standards and EU pesticide Database 
Table 6. Concentrations of Dichlorvos in cabbages around Manzini.

\begin{tabular}{lll}
\hline Sample & $\begin{array}{l}\text { Conc. }(\mathbf{m g} / \mathbf{k g} \text { of wet } \\
\text { sample) } \pm \text { SD }\end{array}$ & $\begin{array}{l}\text { Conc. }(\mathrm{mg} / \mathbf{k g} \text { of dry } \\
\text { sample }) \pm \text { SD }\end{array}$ \\
\hline PHAC1 & $0.371 \pm 0.055$ & $4.372 \pm 0.653$ \\
PHAC3 & $0.112 \pm 0.043$ & $1.280 \pm 0.488$ \\
MATC4 & $0.261 \pm 0.087$ & $2.691 \pm 0.894$ \\
MATC5 & $0.292 \pm 0.036$ & $3.006 \pm 0.368$ \\
NGWC6 & $0.480 \pm 0.080$ & $5.460 \pm 0.911$ \\
NGWC7 & $0.280 \pm 0.146$ & $3.109 \pm 1.622$ \\
NGWC9 & $0.263 \pm 0.000$ & $2.782 \pm 0.000$ \\
MGAC26 & $0.038 \pm 0.000$ & $0.415 \pm 0.000$ \\
\hline
\end{tabular}

According to data analysed 11 samples $(42.31 \%)$ of the cabbages were contaminated with pesticide residues with dimethoate having a high possibility of contamination. The results suggest that the consumers of cabbages were exposed to high concentrations of some organophosphate pesticides that may cause chronic diseases. Also the presence of more than one pesticide may cause synergistic effects as the maximum residue limits are specified for only one pesticide. Therefore, there is a need for continuous surveillance and monitoring programs for pesticide residues in order to protect end user from indiscriminate exposure to pesticides.

Table 7. Concentration of Dimethoate in cabbage samples around Manzini.

\begin{tabular}{lll}
\hline Sample & $\begin{array}{l}\text { Conc. }(\mathbf{m g} / \mathbf{k g} \text { of wet } \\
\text { sample) } \pm \text { SD }\end{array}$ & $\begin{array}{l}\text { Conc. }(\mathbf{m g} / \mathbf{k g} \text { of dry } \\
\text { sample) } \pm \text { SD }\end{array}$ \\
\hline MATC4 & $1.276 \pm 0.315$ & $13.150 \pm 3.246$ \\
MATC5 & $0.429 \pm 0.241$ & $4.416 \pm 2.475$ \\
NGWC6 & $0.071 \pm 0.009$ & $0.805 \pm 0.107$ \\
NGWC7 & $0.042 \pm 0.016$ & $0.463 \pm 0.177$ \\
NGWC9 & $0.190 \pm 0.090$ & $2.009 \pm 0.948$ \\
VULC11 & $0.053 \pm 0.000$ & $0.666 \pm 0.000$ \\
BUOC13 & $0.008 \pm 0.001$ & $0.106 \pm 0.187$ \\
MGAC25 & $0.159 \pm 0.001$ & $1.566 \pm 0.010$ \\
\hline
\end{tabular}

\subsection{Concentration of Organophosphorus Pesticide Residues in Soil}

Most organophosphate pesticides were not detected in the soil samples from different vegetable farms in Manzini region due to their rapid degradation in the soil compared to organochlorine pesticides. Chlorpyrifos was detected in three (3) soil samples from Buoyane and Ngwempisi areas of Manzini.
Table 8. Concentration of Chlorpyrifos in soil samples in Manzini.

\begin{tabular}{ll}
\hline Sample & Concentration (mg/kg of dry sample) \\
\hline BUOS12 & $2.778 \pm 0.134$ \\
BUOS13 & $0.300 \pm 0.180$ \\
NGWsT9 & $0.356 \pm 0.094$ \\
\hline
\end{tabular}

The highest amount of $2.778 \pm 0.134 \mathrm{mg} / \mathrm{kg}$ was found in soil from Buoyane (BUOS12) as shown in table 8. The mean concentration of chlorpyrifos in soil in Manzini was $1.145 \pm$ $0.136 \mathrm{mg} / \mathrm{kg}$. The presence of chlorpyrifos in soil can be explained in terms of its lengthy half-life. There is a wide range of half-lives reported in the literature for soil persistence, ranging from a few days to 4 years, depending on application rate, ecosystem type, and various environmental factors. The dissipative half-life is significantly longer in organic soils than mineral soils [27].

As per British International Standards (BIS), permissible limits of chlorpyrifos and malathion in soil are $30 \mathrm{ppb}$ and $190 \mathrm{ppb}$ respectively [28]. Therefore, the toxicity levels of chlorpyrifos were higher than the permissible limit and may pose threat to soil organisms.

\subsection{Health Threat from Consumption of Vegetables (Tomatoes and Cabbages)}

The target hazard quotient (THQs) of the studied pesticides through consumption of tomatoes and cabbages are presented in table 9. Although these vegetables originate from different parts of Manzini region, they are sold randomly in different markets in Eswatini. Therefore, THQ evaluations were based on the average residue concentrations of pesticides in tomatoes and cabbages from Manzini region of Eswatini. In fact, the sources of the products were not considered in the derivation of the THQ. The THQ computations also distinguished adults from children since each group has their respective levels of tolerance and different responses to chemical contaminants. As such the computations in table 9 are for adults only.

In general, all the THQ values for each contaminant from consumption of tomatoes alone was zero since no pesticides were detected in tomatoes suggesting that the associated health hazard with exposure to pesticides is insignificant for adults. The THQ values for dimethoate and dichlorvos that were detected in cabbages were more than one suggesting that there is a significant health hazard associated with exposure to these contaminants via consumption of cabbages from Manzini.

Table 9. THQ to pesticide exposure through consumption of tomatoes and cabbages.

\begin{tabular}{lllll}
\hline Pesticides & Rfd $(\mathbf{m g} / \mathbf{k g} / \mathbf{d a y})$ & THQ $_{\text {tomatos }}$ & THQ $_{\text {cabbages }}$ & THQ $_{\text {te }}$ \\
\hline Adults & & & & 0.000 \\
Chlorpyrifos & $2.0 \times 10^{-2}$ & 0.000 & 0.000 & 0.000 \\
Malathion & $7.0 \times 10^{-2}$ & 0.000 & 0.000 & 22.684 \\
Dimethoate & $2.0 \times 10^{-4}$ & 0.000 & 22.684 & 0.000 \\
Profenofos & $2.0 \times 10^{-3}$ & 0.000 & 0.000 & 9.048 \\
Dichlorvos & $5.0 \times 10^{-4}$ & 0.000 & 9.048 & 31.732 \\
\hline
\end{tabular}

THQ tomatoes - target hazard quotient for tomatoes, THQ cabbages - Target Hazard Quotient for cabbages, THQtc - total Target Hazard Quotient for both tomatoes and cabbages, TTHQ - total Target Hazard Quotient.

Due to additive nature of contaminants, the risk addition hypothesis was adopted to evaluate the total THQ. 
The combined hazard quotient of both tomatoes and cabbages was as well more than one $(>1.00)$ for dimethoate and dichlorvos signifying that the health hazard associated with the consumption of the two vegetables is significantly high with the potential of toxicity. The relative contributions of each pesticide contaminant to the total pesticide THQ from vegetable consumption is shown in figure 10. Dimethoate was the major risk contributor in the study accounting for almost $71.49 \%$ of the THQ followed by dichlorvos with risk contribution of $28.51 \%$. Others such as chlorpyrifos, malathion and profenofos were not risk contributors since they were not detected in both cabbages and tomatoes.

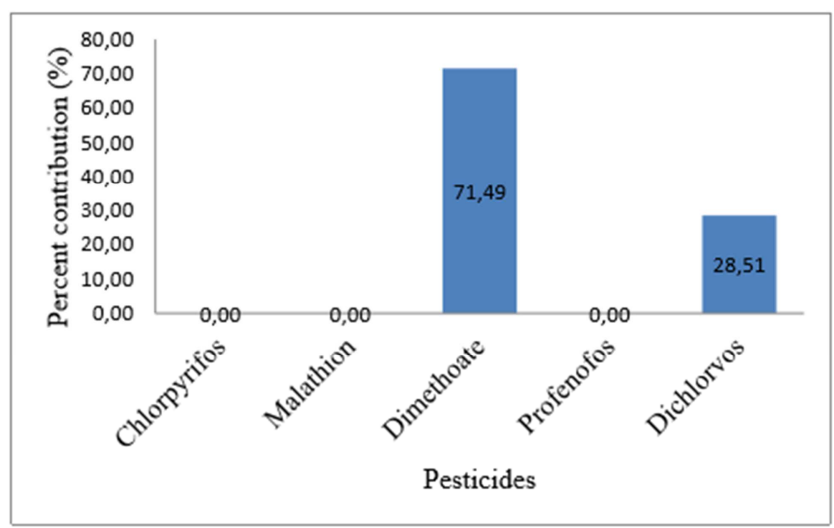

Figure 10. Percentage contribution of each pesticide to the TTHQ.

The study revealed that cabbages from Manzini region contained residues of dichlorvos and dimethoate with residue levels that exceeded EU MRLs and Codex (FAO/WHO) MRLs. Perhaps the reason why pesticides are retained by cabbage is because of their leafy attribute compared to tomatoes which are easy to wash out at irrigation hence, no organophosphate pesticides were detected in tomatoes. Furthermore, the results suggest that the health risk posed by exposure to dimethoate and dichlorvos pesticides to Emaswati populace through consumption of contaminated cabbages estimated by the target hazard quotient is significantly high. The THQs values for these pesticides were more than one (THQ> 1.00). The relative contribution of dimethoate to the total THQ was high accounting for $71.49 \%$.

\section{Conclusions}

The use of pesticides in controlling different types of pests is one of the essential measures of modern food crop production. However, the residues resulting from misapplication of pesticides in vegetables is of crucial concern not only to the people of Eswatini but also the international community at large. In order to benefit from vegetable production, organophosphate pesticides were always applied in excessive dosage and frequency during the growing seasons of vegetables and resulted in food contamination. Indiscriminate use of pesticides and hence the chance of pesticide residue presence in these vegetables and the environment is a matter of great concern. Exposure of farmers and consumers to pesticides is a major threat. The results in this study provide important information on the current contamination status of tomatoes and cabbages in Manzini region and point to the need of taking further measures to control the misuse of some OPPs such as dichlorvos, dimethoate and chlorpyrifos. The government should therefore enforce routine pesticide monitoring programmes, engage suppliers in collection of used pesticide containers from the farmers and deploy more agricultural extension officers to educate farmers on safe use and dangers of pesticides in different vegetable growing areas in Eswatini to prevent, control and reduce environmental pollution as well as residues in food.

\section{Acknowledgements}

The authors are grateful to the Association of Commonwealth Universities (ACU) for the Commonwealth Scholarship that enabled Paul Junior to conduct this study as part of his Masters of Science in Chemistry programme at University of Eswatini.

\section{References}

[1] Sharma, D. R., Thapa, R. B., Manandhar, H. K., Shrestha, S. M. and Pradhan, S. B. (2012). Use of pesticides in Nepal and the impacts on human health and environment. Journal of Agriculture and Environment, 13: 67-68.

[2] Steven, G. (2014). "Organophosphates". Accessed through http://www.toxipedia.org/display/toxipedia/Organophosphates . Retrieved on 7 June 2017.

[3] Jamal, G. A., Hansen, S., Pilkington, A., Buchanan, D., Gillham, R. A. and Abdel-Azis, M. (2002). A clinical neurological, neurophysiological, and neuropsychological study of sheep farmers and dippers exposed to organophosphate pesticides. Journal of Occupational and Environmental Medicine, 59: 434-441.

[4] Eddleston, M., Buckley, N. A., Eyer, P. and Dawson, A. H. (2008). Management of acute organophosphorus pesticide poisoning. Lancet, 371(9612): 597-607.

[5] World Bank (2008). World Development Report on Agriculture for Development. Retrieved from http://siteresources.worldbank.org/INTWDR2008/Resources/ WDR_00_book.pdf.

[6] Dementi, B. (1994). 'Ocular Effects of Organophosphates: A Historical Perspective of Saku Disease'. Journal of Applied Toxicology, 14(2): 119-129.

[7] Andrew, G. R. (2002). Pesticide residue analysis and its relationship to hazard characterization and intake estimation. Pest Management Science, (58): 1073-1082.

[8] Akan, J. C., Jafiya, L., Mohammed, Z. and Abdulrahman, F. I. (2013). Organophosphorus pesticide residues in vegetables and soil samples from Alau dam and Gongulong agricultural areas, Borno State, Nigeria. International Journal of Environmental Monitoring and Analysis, 1(2): 58-64. 
[9] Frederick, A. A. (2011). Assessment of pesticide residues in vegetables at the Farm Gate: Cabbage (Brassica oleracea) Cultivation in Cape Coast, Ghana. Research Journal of Environmental Toxicology, 5: 180-202.

[10] Sekhon, M. K. and Kaur, M. (2004). Role of small farmers in diversification of Punjab agriculture with vegetables. Indian Journal of Agricultural Marketing, 18(1): 80-88.

[11] Thompson, C. F. (2012). Swaziland business year book. Mbabane-Swaziland: Christina Forsyth- Thompson.

[12] NAMBoard (2009). Annual Report for National Agricultural Marketing Board, Manzini, Swaziland.

[13] FAOSTAT (2016). World: Vegetable Total Production, Mbabane, Swaziland.

[14] Frederick, M. and Fishel, K. (2005). Pesticide information office; Florida cooperative extension service, Institute of Food and Agricultural Sciences, University of Florida.

[15] EU Pesticide Database. Available http://ec.europa.eu/food/plant/pesticides/eu-pesticidesdatabase/public/?event=pesticide-residuecurrentMRL \$language=EN. Accessed on $13^{\text {th }}$ February, 2018.

[16] Codex Alimentarius International Food Standards. Food and Agriculture Organisation of United Nations and World Health Organisation. http://www.fao.org/fao-whocodexalimentarius/codex-texts/dbs/pestres/pesticides/en. Accessed on $8^{\text {th }}$ February, 2018.

[17] Singh, A. S., Mhlanga, N. P. and Kibirige, D. (2017). Gender contribution on profitability of vegetable production in the rural Manzini region of Swaziland. International Journal of Research in Management, Economics and Commerce, 7(6): 90-98.

[18] European commission (EC). Establishing community methods of sampling for the official control of Pesticide Residues in and on products of plant and animal origin and Repealing Directive. OJEUL 2002, 187, 30-43.

[19] Anastassiades, M., Lehotay, S. J., Štajnbaher, D. and Schenck, F. J. (2003). Fast and easy multiresidue method employing acetonitrile extraction/partitioning and "dispersive solid phase extraction" for the determination of pesticide residues in produce. International Journal of Association of Official Analytical Chemists, 86: 412-431.
[20] Prodhan, M. D. H., Papadakis, E. N. and Mourkidou, E. P. (2015). Determination of multiple pesticide residues in eggplant with liquid chromatography-mass spectrometry. Food Analytical Methods, 8: 229-235.

[21] U.S. EPA. (1996). Integrated risk information system. United States Environmental Protection Agency. Office of Health and Environmental Assessment, Washington, D. C.

[22] Donkor, A., Osei-Fosu, P., Nyarko, S., Kingsford-Adaboh, R. and Okyere, J. Y. A. (2015). Health Risk Assessment of Pesticide Residues via Dietary intake of cowpeas and Bambara beans among adults in Accra Metropolis, Ghana. Research Journal of Chemical and Environmental Science, 3 (1): 10-18.

[23] Bempah, C. K., Donkor, A., Yeboah, P. O., Dubey, B. and Osei-Fosu, P. (2011). A Preliminary assessment of consumer's exposure to organochlorine pesticides in fruits and vegetables and the potential health risk in Accra Metropolis, Ghana. Food Chemistry, 128(4): 1058-1065.

[24] Chien, L. C., Hung T. C., Choang, K. Y. and Meng, P. J. (2002). Daily intake of TBT, $\mathrm{Cu}, \mathrm{Zn}, \mathrm{Cd}$ and As for fishermen in Taiwan. Science of the Total Environment, 285: 177-185.

[25] Xaba, B. G. and Masuku, M. B. (2013). An analysis of the vegetable supply chain in Swaziland. Sustainable Agriculture Research; Vol. 2, No. 2. Published by Canadian Center of Science and Education. http://dx.doi.org/10.5539/sar.v2n2p1.

[26] Eurachem Guide (2014). The Fitness for Purpose of Analytical Methods - A Laboratory Guide to Method Validation and Related Topics, $2^{\text {nd }}$ edition, Magnusson, B. and Ornemark, U. (eds.). www.eurachem.org.

[27] Gebremariam, S. Y., Beutel, M. W., Yonge, D. R., Flury, M. and Harsh, J. B. (2012). Adsorption and desorption of chlorpyrifos to soils and sediments. Reviews of Environmental Contamination and Toxicology, 215: 123-175.

[28] Baride, M. V., Patil, S. N. and Golekar, R. (2015). Estimation of pesticide residues in soil, ground water, vegetables and fruits: A case study of Jalgaon district, Maharashtra (India). SRTM University's Research Journal of Science. Spl. Vol. No. 1 (ISSN: 2227-8594). 